\title{
DTI Values in Key White Matter Tracts from Infancy through Adolescence
}

\author{
A. Cancelliere, F.T. Mangano, E.L. Air, B.V. Jones, M. Altaye, A. Rajagopal, S.K. Holland, D.A. Hertzler II, and W. Yuan
}

\begin{abstract}
BACKGROUND AND PURPOSE: DTI is an advanced neuroimaging technique that allows in vivo quantification of water diffusion properties as surrogate markers of the integrity of WM microstructure. In our study, we investigated normative data from a large number of pediatric and adolescent participants to examine the developmental trends in DTI during this conspicuous WM maturation period.
\end{abstract}

MATERIALS AND METHODS: DTI data in 202 healthy pediatric and adolescent participants were analyzed retrospectively. Fractional anisotropy and mean diffusivity values in the corpus callosum and internal capsule were fitted to an exponential regression model to delineate age-dependent maturational changes across the WM structures.

RESULTS: The DTI metrics demonstrated characteristic exponential patterns of progression during development and conspicuous agedependent changes in the first 36 months, with rostral WM tracts experiencing the highest slope of the exponential function. In contrast, the highest final FA and lowest MD values were detected in the splenium of the corpus callosum and the posterior limb of the internal capsule.

CONCLUSIONS: Our analysis shows that the more caudal portions of the corpus callosum and internal capsule begin the maturation process earlier than the rostral regions, but the rostral regions develop at a more accelerated pace, which may suggest that rostral regions rely on development of more caudal brain regions to instigate their development. Our normative DTI can be used as a reference to study normal spatiotemporal developmental profiles in the WM and help identify abnormal WM structures in patient populations.

ABBREVIATIONS: ALIC = anterior limb of internal capsule; $\mathrm{CC}=$ corpus callosum; $\mathrm{FA}=$ fractional anisotropy; $\mathrm{MD}=$ mean diffusivity; $\mathrm{PLIC}=$ posterior limb of internal capsule

T he behavioral, cognitive, and biologic changes associated with brain development and its aberrations remain poorly understood. The development and maturation of WM tracts during childhood and adolescence are intimately associated with these processes. ${ }^{1,2}$ DTI has allowed quantification of the magnitude and direction of water diffusivity in the cerebral WM and, thus, the ability to investigate its development in better detail. Considerable evidence has shown that the diffusion of water in the human brain is a dynamic process, changing with brain maturation and

Received May 4, 2012; accepted after revision July 31.

From the Division of Pediatric Neurosurgery (A.C., F.T.M., E.L.A., D.A.H. II); Department of Radiology, Imaging Research Center (B.V.J., A.R., S.K.H., W.Y.); and Division of Biostatistics and Epidemiology (M.A.), Cincinnati Children's Hospital Medical Center and University of Cincinnati College of Medicine, Cincinnati, Ohio; and Department of Emergency Medicine, Harvard-Affiliated Emergency Medicine Residency, Beth Israel Deaconess Medical Center, Boston, Massachusetts (A.C.).

Please address correspondence to Weihong Yuan, PhD, Pediatric Neuroimaging Research Consortium/Imaging Research Center, Cincinnati Children's Hospital, MLC 5033, 3333 Burnet Ave, Cincinnati, OH 45229; e-mail: Weihong.Yuan@ cchmc.org

http://dx.doi.org/10.3174/ajnr.A3350 structural integrity of WM. The emerging consensus is that the developing WM is characterized by intrinsic modifications, including an increase in the number of its constituent macromolecules and cytoskeletal microfilaments, and the extrinsic modification of axon caliber resulting in displacement and decrease in brain-water content. ${ }^{3,4}$ In addition, constraining changes in tissue macrostructure, including increased WM packing and the activity of sodium channels, have been postulated to play a key role in increasing the nonrandom diffusion of water, or anisotropy. ${ }^{4-7}$ These studies have demonstrated a positive correlation between the preferential directionality of water diffusion along WM tracts and age, whereas a negative correlation is demonstrated between the magnitude of water diffusion in specific WM tracts and age. ${ }^{6}$ The most widely adopted scalar DTI metrics to quantify these events are FA, which quantifies the degree of preferential restriction in water diffusion, and $\mathrm{MD}$, which measures the overall magnitude of water diffusion. The quantification of these parameters by DTI has enabled clinicians to construct spatial representations of brain WM structures and to carefully examine their microarchitecture in the developing brain ${ }^{4,8-10}$ and the mature brain. ${ }^{11-13}$ 
Along with an improved detection of age-related changes in water diffusion-based properties of WM tracts, recent studies have postulated that DTI may be clinically useful in evaluating brain pathologic findings and the subsequent therapeutic response by comparing WM diffusion changes with normative values. ${ }^{14-16}$ Previous DTI studies of WM development have been limited by small numbers of pediatric patients, inclusion of data from multiple institutions, combination of adult and pediatric data, or inclusion of potentially biasing pathologies without further follow-up. $4,8,13,17-22$

The aim of our study was, thus, to report a representative dataset of normal age-related changes in FA and MD values detected in the developing brain, derived from 202 healthy participants ranging in age from birth to adolescence. We propose that these normative data represent true age-appropriate DTI values during the development of susceptible and critical WM tracts. The details of these developmental processes are crucial to better understand the mechanisms of WM development, maturation, and its aberrancies caused by various neurologic insults.

\section{MATERIALS AND METHODS \\ Study Population}

The institutional review board of Cincinnati Children's Hospital Medical Center approved this study. We conducted a retrospective chart review of normal MR imaging scan results presenting from 2003 until 2008. Inclusion criteria were 1) between ages 0 (full-term) and 18 years, 2) normal brain MR imaging results, 3) no history of neurologic disease, and 4) no development of neurologic disease at a minimum of 4 months after the initial scan. A total of 202 participants (age range, 0.2-215.4 months) met the inclusion criteria, and their DTI data were analyzed. The distribution of the number of participants per age in months is reported in Fig $1 D$.

Children younger than 5 years required either sedation or anesthesia during the clinical scans because of their young age. The DTI data were carefully reviewed. Datasets that had signal dropout because of head movement or Nyquist ghost artifacts because of the eddy current were discarded.

\section{Image Acquisition and Data Processing}

We acquired all of the MR imaging and DTI data for all 202 participants by using 1.5T Signa Horizon LX scanners (GE Healthcare, Milwaukee, Wisconsin). Scanning parameters for DTI sequences were the same for all 202 participants and consisted of diffusion-weighted, single-shot spin-echo EPI in the axial plane with a b-value of $1000 \mathrm{~s} / \mathrm{mm}^{2}$, a TR of 12,000 ms, a TE of 73-93 $\mathrm{ms}$, and 15 diffusion directions. All examinations met the following resolution specifications: FOV, $384 \times 384 \mathrm{~mm}^{2}$; acquisition matrix, $128 \times 128$; and resolution, $3 \times 3 \times 3 \mathrm{~mm}^{3}$. Imaging data were reconstructed with a matrix of $256 \times 256$, resulting in a reconstructed in-plane resolution of $1.5 \times 1.5 \mathrm{~mm}^{2}$.

DTI Studio 2.4 software (Johns Hopkins University, Baltimore, Maryland; lbam.med.jhmi.edu) was used for image reconstruction, postprocessing, and region-of-interest data analysis. ${ }^{8}$ On a pixel-bypixel basis, 6 elements (Dxx, Dyy, Dzz, Dxy, Dxz, and Dyz) were calculated and were diagonalized to calculate the 3 eigenvalues $\left(\lambda_{1}\right.$, $\lambda_{2}, \lambda_{3}$ ) corresponding to the 3 eigenvectors in the diffusion tensor matrix. We calculated FA and MD values using standard methods. ${ }^{23}$
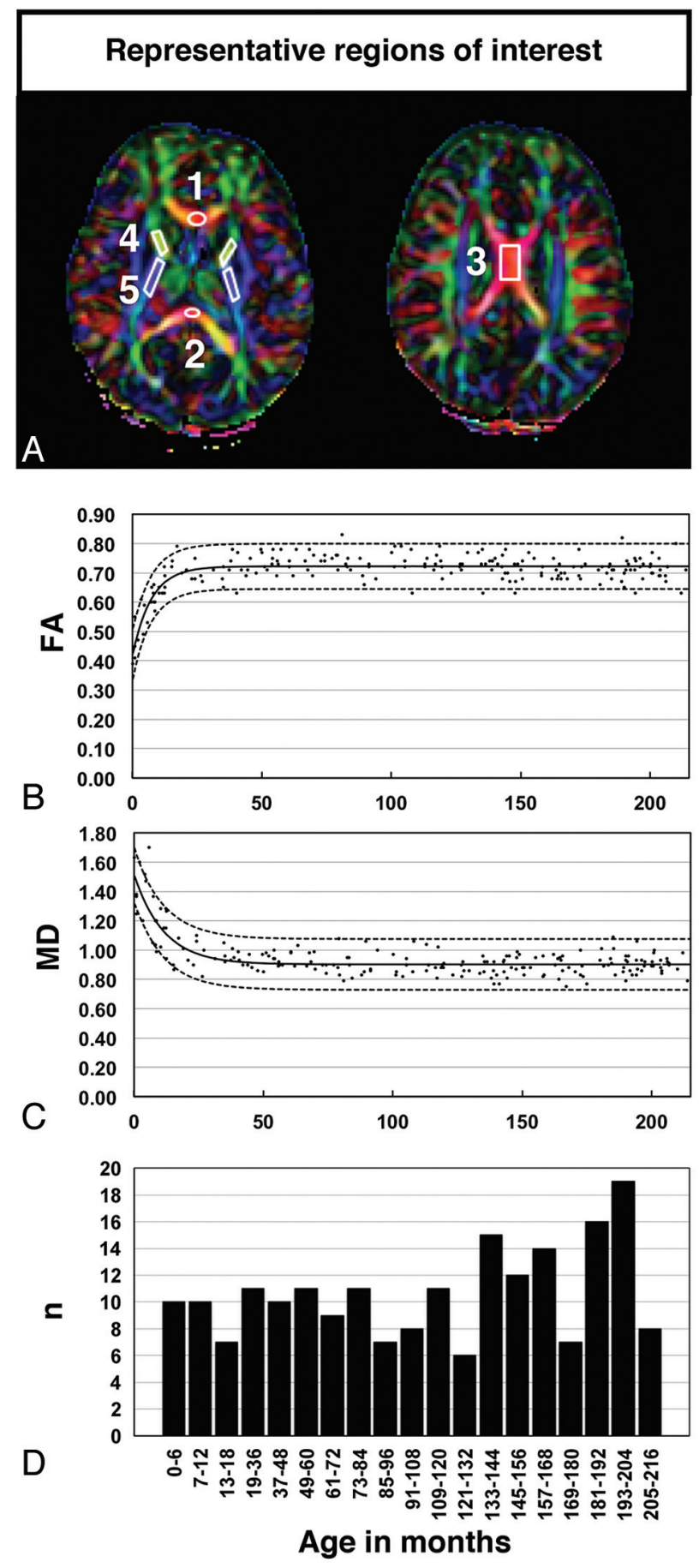

FIG 1. A, Selection of the ROls overlaid on representative colorcoded FA images. ROls were selected in the following regions: 1, genu of the CC; 2 , splenium of the CC; 3 , body of the CC; 4 , ALIC; and 5 , PLIC. B-C, Best-fit exponential functions (solid lines) and the respective $95 \%$ prediction intervals (dashed lines) overlaid on the scatterplots derived from the raw data of FA and MD values, respectively, detected in the gCC from individual participants. $D$, The large number of participants throughout the entire study, including the period of maximal changes, is representative of normative data. Abbreviations: $n=$ number of participants.

\section{Selection of Regions of Interest}

A color-coded FA map was used to define the 5 ROIs analyzed in this study (Fig 1A): genu of the CC, body of the CC, splenium of the CC, ALIC, and PLIC. Given that no significant differences 
Table 1: Age-dependent changes in FA and MD in cerebral WM of the developing brain with an exponential regression model

\begin{tabular}{|c|c|c|c|c|c|c|}
\hline \multirow[b]{2}{*}{ Region } & \multicolumn{4}{|c|}{ Regression Coefficients } & \multicolumn{2}{|c|}{ Exponential Fit Model } \\
\hline & a & b & c & $y$-intercept & $r^{2}$ & RMSE \\
\hline \multicolumn{7}{|l|}{ FA } \\
\hline gCC & -0.307 & 0.138 & 0.722 & 0.416 & 0.629 & 0.039 \\
\hline bcc & -0.327 & 0.109 & 0.740 & 0.414 & 0.562 & 0.046 \\
\hline $\mathrm{sCC}$ & -0.365 & 0.117 & 0.785 & 0.420 & 0.729 & 0.039 \\
\hline ALIC & -0.286 & 0.125 & 0.565 & 0.279 & 0.745 & 0.029 \\
\hline PLIC & -0.206 & 0.073 & 0.655 & 0.449 & 0.772 & 0.023 \\
\hline \multicolumn{7}{|l|}{ MD } \\
\hline gCC & 0.616 & 0.088 & 0.902 & 1.518 & 0.659 & 0.088 \\
\hline bcc & 0.617 & 0.096 & 0.818 & 1.435 & 0.686 & 0.070 \\
\hline $\mathrm{sCC}$ & 0.857 & 0.069 & 0.831 & 1.688 & 0.671 & 0.129 \\
\hline ALIC & 0.429 & 0.134 & 0.771 & 1.200 & 0.683 & 0.049 \\
\hline PLIC & 0.319 & 0.089 & 0.732 & 1.051 & 0.692 & 0.042 \\
\hline
\end{tabular}

Note:-Given are the values for the regression coefficients describing the best-fit function of the FA and MD values for each region of interest.

bCC indicates body of the corpus callosum; gCC, genu of the CC; $r^{2}$, square of the correlation coefficient, describing the goodness of fit; RMSE, root mean square error; sCC, splenium of the CC.

were noted between the 2 hemispheres, we averaged the right and left values for analysis in ALIC and PLIC. The method is similar to the approach of Hermoye et $\mathrm{al}^{8}$ and has also been described in our past studies. ${ }^{15,24}$ Each region of interest was placed manually by a single investigator (W.Y.), with the assistance of a neuroradiologist (B.V.J.) and a pediatric neurosurgeon (F.T.M.). There was not an optimal region-of-interest size that fit all participants with such a wide age range as well as individual anatomic variation. Thus, the following analysis was performed to assess whether the region-of-interest size had a significant effect on the results: After examining the region-of-interest size data plotted against age, as expected, we detected a linear positive correlation between region-of-interest size and age in some ROIs. We removed this agedependent increase by fitting a linear regression model relating size with age. The residual difference between fitted and regionof-interest size was then extracted. We then conducted a correlation analysis between the residual from the DTI vs age regression and the residual region-of-interest size. No significant correlation was found at a $P$ level of .05 for any region of interest for either FA or MD. Therefore, the region-of-interest size was discarded as a potential confounding factor in the analysis.

To evaluate the consistency of region-of-interest selection, we performed intrarater reliability (test-retest reliability) on 30 datasets (a subset of the overall 202 participants) and the same operator (W.Y.) repeated the test 5 times, each at least 3 days apart. The intraclass correlation coefficients for intrarater reliability of measurements were very consistent for all ROIs (intraclass correlation coefficient, $0.96,0.91,0.82$, and 0.81 for FA in the genu of the CC, splenium of the CC, PLIC, and ALIC, respectively). We also tested 2 additional raters to assess the interrater conformity by using the same subset of 30 participants. The ICC for interrater reliability of measurements was also very consistent for all ROIs, in the range of $0.73-0.90$.

\section{Statistical Analysis}

The program, Statistical Analysis Software (SAS, V9.2, SAS Institute, Cary, North Carolina), was used for statistical analysis. Matlab 7.1 (MathWorks, Natick, Massachusetts) was used to establish a normative range of DTI values in our ROIs with 95\% prediction intervals (Table 1). To investigate the influence of age on the development of FA and MD, we further performed regres- sion analysis. These diffusion parameters were plotted against age. After comparing all potential best-fit functions, we chose an exponential pattern, which optimally described all ROIs investigated and resulted in the highest $r^{2}$ values (Table 1). The curve fitting to a monoexponential model was performed with use of the cftool function in Matlab 7.1 (Fig 1B). Equation 1 describes the exponential best-fit function, wherein the b-parameter represents the time constant of the exponential curve, $x$ represents the participant's age in months, $c$ represents the value at which $x$ reaches $\mathrm{x}_{\infty}$ (maximal value for the corresponding DTI parameter), and $c+a$ represents the $y$-intercept of the function. The parameter estimate for $a, b$, and $c$ for FA and MD obtained from the exponential models are given in Table 1.

$$
\text { 1) DTI parameters }(F A \text { or } M D)=a^{\star} e^{\left(-b^{*} x\right)}+c
$$

The fitted model in Equation 1 contains 2 distinct trends: a curvilinear part and a relatively flat part. We identified the age in months wherein the fitted value of the curve from this model reaches the flat line. To statistically compare the differences between different regions of interest, we split the data into the curvilinear part and the flat part. We analyzed the first part of the data by using a linear fit on log-transformed data to fit the following regression line:

$$
\begin{aligned}
& \text { 2) DTI parameter }(F A \text { or } M D)=\beta_{0}+\beta_{1} \text { age } \\
& \text { in months }+\beta_{2} R O I+\beta_{3} \text { age in months }{ }^{*} \mathrm{ROI}
\end{aligned}
$$

To test if the 2 slopes (generated from fitting the FA or MD against age in months for any 2 regions of interest) were parallel or different, we tested the significance of the interaction term $\left(\beta_{3}\right)$ from Equation 2 (Table 2). We also evaluated the flat part of the curve by comparing the mean of the DTI data during the age range corresponding to the flat curve of the exponential model between any 2 regions of interest (Table 3 ).

\section{RESULTS}

The data were grouped according to the WM fiber regions that were analyzed, namely the CC or the internal capsule. A representative example of the fitted exponential curves for FA and MD values juxtaposed on the raw data are shown in Fig $1 B$. The $y-$ intercepts of the FA and MD curves in the relative ROIs were first 
Table 2: Statistical comparison among b-parameters between regions

\begin{tabular}{lllllr}
\hline DTI Parameter & Comparison & $\boldsymbol{\beta}_{\mathbf{3}}$ & SE $\left(\boldsymbol{\beta}_{\mathbf{3}}\right)$ & $\boldsymbol{t}$ & $\boldsymbol{P}$ Value \\
\hline FA & gCC vs bCC & 0.0114 & 0.0100 & 1.16 & .251 \\
& gCC vs sCC & 0.0121 & 0.0082 & 1.48 & .142 \\
& bCC vs SCC & 0.0007 & 0.0010 & 0.07 & .941 \\
MD & ALIC vs PLIC & 0.0163 & 0.0042 & 3.89 & $<.001$ \\
& gCC vs bCC & -0.0042 & 0.0171 & -0.25 & .805 \\
& gCC vs SCC & -0.0423 & 0.0173 & -2.45 & .015 \\
& bCC vs SCC & -0.0381 & 0.0212 & -1.79 & .076 \\
& ALIC vs PLIC & -0.0238 & 0.0084 & -2.85 & .005 \\
\hline
\end{tabular}

Note:- The gCC and sCC are statistically different for MD. The age-dependent changes in FA and MD are statistically different between the ALIC and PLIC.

$\beta_{3}$ indicates an interaction term; SE, standard error; $t, t$ value; $\left(\beta_{3} / \mathrm{SE}\left(\beta_{3}\right) ; \mathrm{gCC}\right.$, genu of the $\mathrm{CC} ; \mathrm{bCC}$, body of the $c C$; sCC, splenium of the cc.

Table 3: Statistical comparison among c-parameters between regions

\begin{tabular}{lcclcc}
\hline DTI Parameter & Region & Mean \pm SD & Comparison & Mean Difference & $\boldsymbol{P}$ Value \\
\hline FA & gCC & $0.723 \pm 0.038$ & gCC vs bCC & -0.015 & .0015 \\
& bCC & $0.738 \pm 0.043$ & gCCvs sCC & -0.063 & .0001 \\
& SCC & $0.786 \pm 0.038$ & bCCVs sCC & -0.048 & .0001 \\
& ALIC & $0.565 \pm 0.026$ & ALIC vs PLIC & -0.092 & .0001 \\
MD & PLIC & $0.657 \pm 0.023$ & & & \\
& gCC & $0.897 \pm 0.068$ & gCC vs bCC & 0.082 & .0001 \\
& bCC & $0.815 \pm 0.056$ & gCC vs sCC & 0.083 & .0001 \\
& SCC & $0.814 \pm 0.090$ & bCC vs sCC & 0.001 & .9163 \\
& ALIC & $0.770 \pm 0.041$ & ALIC vs PLIC & 0.043 & .0001 \\
& PLIC & $0.727 \pm 0.034$ & & & \\
\hline
\end{tabular}

Note:- gCC indicates genu of the CC; bCC, body of the CC; sCC, splenium of the sCC.

analyzed to gain insight into interregional differences present at birth. The y-intercepts of the FA fitted curves in the genu of the CC, body of the CC, and splenium of the CC did not appear to be different $(0.416,0.414$, and 0.420, respectively; Fig 2). However, the $y$-intercept of the MD fitted curve detected in the splenium of the CC was higher compared with the genu of the CC and body of the CC (1.688, 1.518, and 1.435, respectively, Table 1$)$. In the internal capsule, the $y$-intercept of the FA fitted curve of the ALIC was lower than that of the PLIC ( 0.279 and 0.449 , respectively), whereas the $y$-intercept of the MD of the ALIC was higher compared with that of the PLIC (1.200 and 1.051, respectively).

We then examined the b-parameter values from the exponential model to compare the rate of change in FA and MD in relationship to age in months across regions (Table 1). The most conspicuous age-dependent changes were detected in the first 24-36 months of age in all ROIs (Fig 3). The values of the bparameter for FA in the genu of the CC and ALIC were higher compared with their respective caudal regions $(0.138,0.109$, and 0.117 in the genu of the $\mathrm{CC}$, body of the $\mathrm{CC}$, and splenium of the CC, respectively; and 0.125 and 0.073 in the ALIC and PLIC, respectively; Fig 2). When the MD in the CC was analyzed, the lowest b-parameter values were detected in the splenium of the CC and PLIC compared with more rostral regions $(0.069,0.088$, and 0.096 in the splenium of the CC, genu of the CC, and body of the CC, respectively; and 0.089 and 0.134 in the PLIC and ALIC, respectively; Table 1). The DTI data were split into 2 parts to determine the statistical differences, one that progressively increases with age in months and a relatively stable flat portion. The $\beta_{3}$ (from Equation 2), which directly tests the statistical difference between the slopes, was significantly different for MD between the genu of the CC and splenium of the CC, whereas it showed a trend for FA between the genu of the CC and splenium of the CC ( $\beta_{3}$
$P=.015$ and $P=.142$, respectively). In contrast, the $\beta_{3}$ for both FA and MD was significantly different between the ALIC and PLIC $(P<.001$ and $P<.005$, respectively, Table 2$)$.

We then compared the magnitude of the c-parameter values and the age in months at which the c-parameter is reached. In the CC, the highest c-parameter value of the FA curve in the CC was detected in the splenium of the CC, whereas the PLIC was higher compared with the ALIC (Fig 3). For the MD fitted curves, the splenium of the CC and body of the CC and PLIC cparameter values were lower compared with the more rostral ROIs. It is interesting to note that the longest-lasting increases in FA were detected in the PLIC (at 83 months) and in the MD in the splenium of the CC (at 129 months; Fig 3). For the flat part of the curve, the difference in the mean between the 2 regions of interest was statistically significant, except for the difference between the body of the CC and splenium of the CC values for the MD (Table 3).

\section{DISCUSSION}

The use of DTI as a tool to investigate the development of WM tracts and help clinicians outline structural abnormalities is currently limited by the availability of a normative DTI data base. To the best of our knowledge, our study is the first to report FA and MD values with more than 200 participants in a large age range, with DTI data acquired at a single institution by homogeneous sequence parameters. Moreover, the age of our participants includes the entire period in which the largest age-dependent changes are detected, thus more accurately reflecting normative DTI measurements during this most crucial period.

\section{Differences in Developmental Timing and Regional Pattern of Maturation}

The changes in DTI parameters were investigated to gain insight into intraregional and interregional developmental changes in the major WM tracts and infer details about myelination and axonal development. We chose to specifically focus on the CC and internal capsule, as these regions provide massive interhemispheric connections between homologous neocortical and corticospinal areas, serving a crucial role in sensorimotor integration, attention, memory, and general cognitive functioning. Moreover, they are among the most susceptible to hypoxiaischemia and hydrocephalus, the most common condition treated in the pediatric neurosurgical population.

The changes in WM diffusion indices occur along a predictable and quantifiable temporal profile, described by our empirically derived exponential model. Our results showed that the FA and MD values of the PLIC were lower during the neonatal period compared with those of the ALIC.

However, we were not able to statistically compare these differences in these DTI indices because of the paucity of data at 

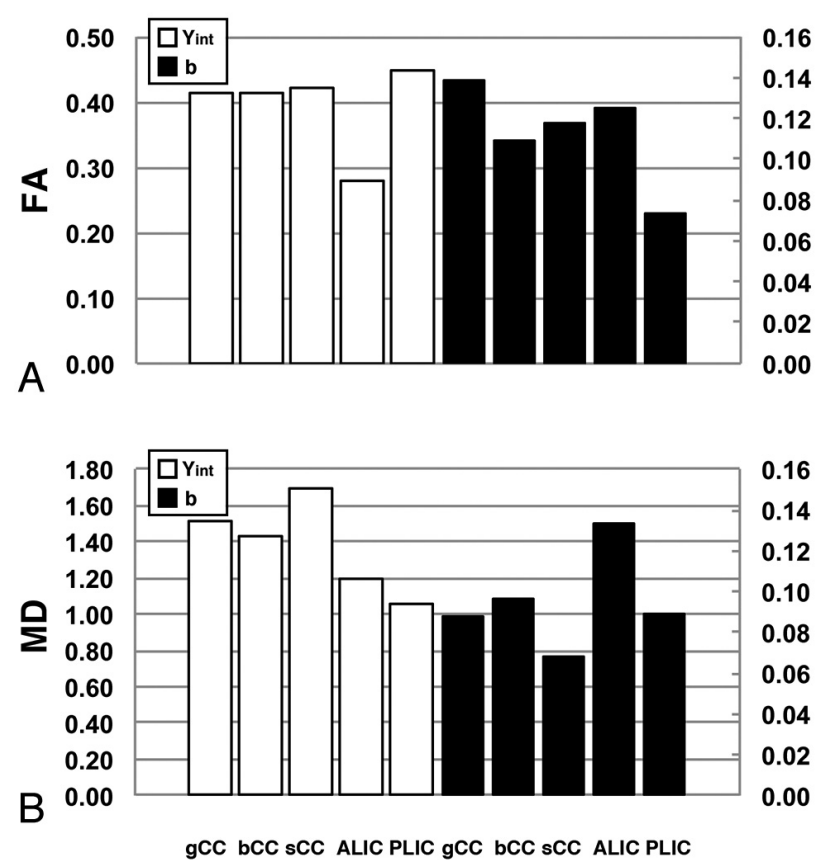

FIG 2. Regression analysis of FA and MD values at approximately the neonatal period and early in development. $A$, Graphic comparison of the $y$-intercepts reveals a trend toward the anterior regions of the internal capsule having lower anisotropy and higher mean diffusivity at approximately the neonatal period. B, Comparison of the b-parameter values of the FA and MD best-fit functions suggests that the rostral regions undergo more rapid, age-dependent changes compared with the caudal regions in each respective region of interest.

these time points. Given this limitation, research efforts toward the recruitment of representative datasets of healthy WM during the neonate period are ongoing at our institution. Such studies would clearly provide novel insight into the significant differences in FA and MD values in these ROIs, thus allowing us to infer important details about white matter development, and maturation in neonates. Nonetheless, our data are consistent with several postmortem studies ${ }^{19,25-29}$ investigating the extent of myelination, which have reported that the PLIC is an "early" initiator of myelination, which begins before birth compared with other WM structures ${ }^{19,25-27}$ and that the first myelinated fibers appear in the ninth fetal month in the lenticulothalamic region, whereas myelination of the fibers in the ALIC lags behind that of the PLIC. ${ }^{28,29}$ Our data are also consistent with previous findings ${ }^{21,22,25,29,30}$ demonstrating an initial rapid, robust increase in WM FA and a concomitant decrease in MD in the first 24 to 36 months of life (Fig 3). Close comparison of the b-parameter value suggests that each region of interest develops at a different rate (Table 1). Despite a trend of the greatest rate of age-dependent changes occurring in FA in rostral ROIs, statistically significant differences were noted only between the ALIC and PLIC and in the MD between the genu of the CC and splenium of the CC. These results detected in the CC and internal capsule also correlate with other studies $^{19,28,31,32}$ demonstrating a conspicuous acceleration in myelination in the anterior regions of the respective WM tracts from the fourth to the eighth postnatal month.

The final FA and MD values of the rostral regions both occurred at earlier time points and were lower, and higher, respectively compared with caudal ROIs (Figs 2 and 3). These data sug- gest that though age-dependent changes in anisotropy are significantly higher during early childhood in the PLIC compared with the ALIC and are similar in the CC, the rostral regions of the CC and internal capsule develop more rapidly as time progresses. In agreement with previous studies, ${ }^{18,19,21}$ the posterior regions of these tracts attain on overall higher degree of anisotropy and lower diffusivity and continue to undergo changes later in development. The relationship between changes in these diffusion properties and corresponding behavioral, cognitive, and sensorimotor modalities are still being uncovered. ${ }^{2}$ Consistent with our findings, an intriguing idea is that these physiologic responses are the result of the environmental acquisition of more complex cognitive, executive, and visual spatial tasks that occur during childhood and adolescence, thus requiring the recruitment of more anterior structures. At present, the increases in integrity and organization of neurofilaments, microtubules, axonal diameter, layers of myelination, dendritic arborization and synaptogenesis, and structural WM fiber tract attenuation and coherence have been invoked as a cause of the observed increase in anisotropy, though a definitive answer has yet to be conclusively shown. ${ }^{5,22,33-35}$ The prevailing consensus in the field is that increases in FA are associated with a decrease in brain-water content and correlate with concomitant WM organization, myelination, and maturation. . $4,8,18,21,22,29,36,37$ In contrast, the decrease in MD values correlates with decreases in the volume of extracellular space, intracellular macromolecular concentration, and total brain-water content. ${ }^{21,29}$ Recent studies by our research group $^{38,39}$ have shown that DTI changes are associated with myelin loss and cytopathologic disruption in an animal model of induced hydrocephalus. Thus, we can assume that the changes in FA reflect these processes and are associated with structural changes occurring in WM bundles during the development of the myelin sheath. These assumptions are in line with the idea that tracts emanating from more anterior gray matter structures undergo more rapid developmental changes and reach maturation earlier, whereas more posterior regions may be undergoing more prolonged and robust myelination and organization.

Our study enabled us to compare the interregional differences in the patterns of normal development of the WM tracts. Our aim was to provide normative data in DTI indices by using healthy participants without any known neurologic disease. It was expected that a large sample size minimizes the effect of individual outliers on the conclusions. However, some of the results did not reach statistical significance partially because of the cross-sectional nature of our study. Future studies with longitudinal design would be better suited to determine and compare the individual progression of white matter development and maturation. A longitudinal study design also would be beneficial to investigate the effects of individual differences in maturation patterns as well as WM aberrations after traumatic injuries or other leukoencephalopathic conditions. By including a larger number of data points during the neonatal age range, critical insight would be provided regarding WM changes at this developmental period. However, these limitations would be beyond the scope of our present study. Future longitudinal studies will further our understanding of both interindividual physiologic differences and pathologic states, which are currently lacking by our study design. The clin- 
FA

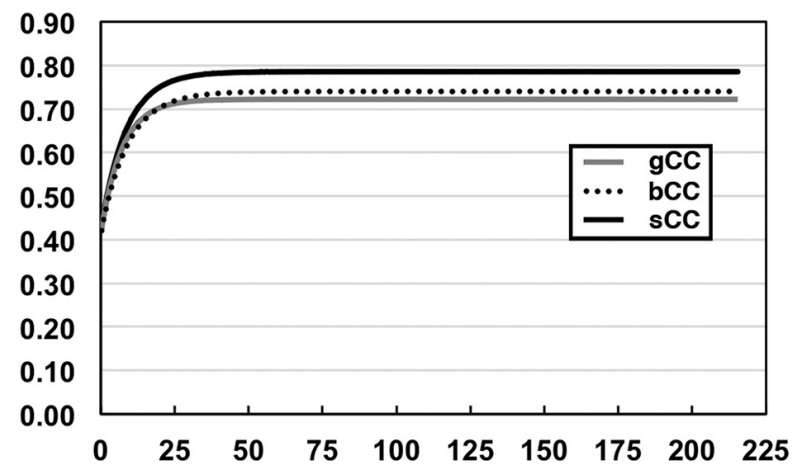

A

FA

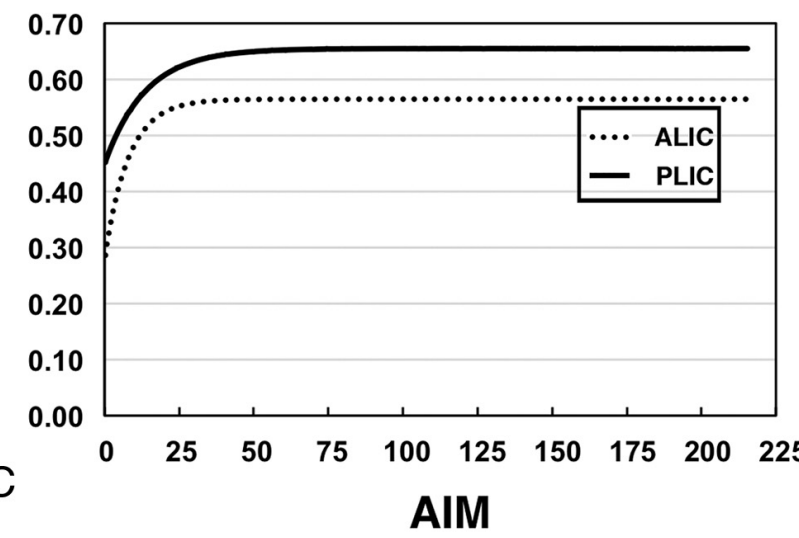

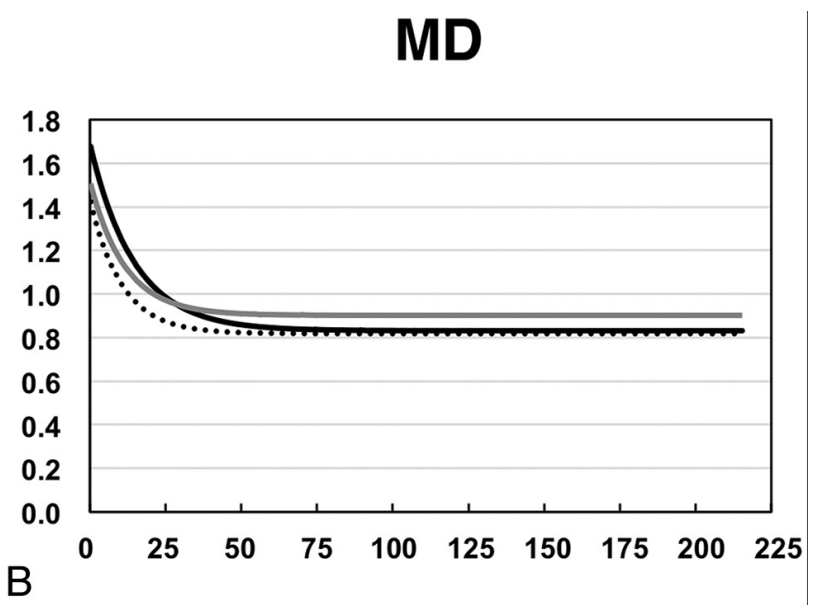

MD

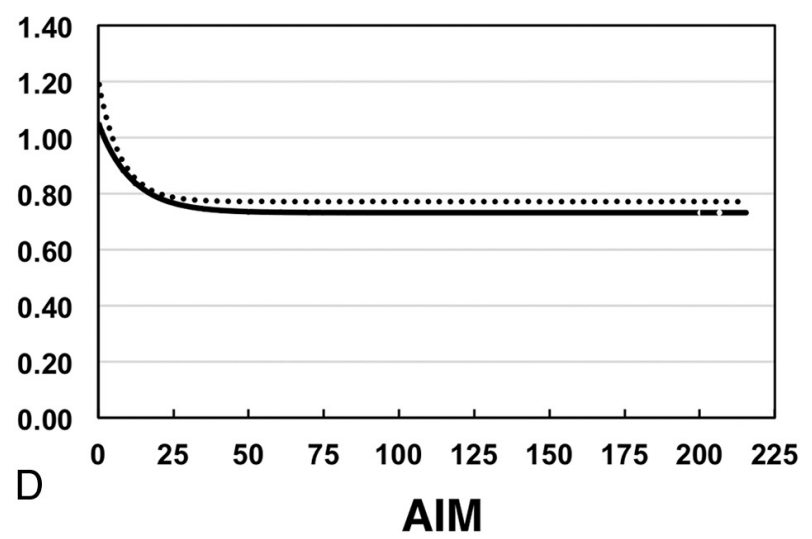

FIG 3. Interregional comparison of fitted exponential curves of FA and MD values. These data are fitted to a monoexponential model with 3 fitted parameter values, shown in Table 1; AlM indicates age in months.

ical potential of using water molecules as a probe to assess neuronal structural integrity and microscopic detail will continue to grow as we learn more about the true meaning of anisotropy and diffusivity.

\section{CONCLUSIONS}

Our study presents the evolution of brain-water anisotropic diffusion parameters in 202 participants between term birth and adolescence. Our data are consistent with the notion that more anterior regions, which are developmentally more immature at birth, have a faster rate of development between birth and 24 months, whereas more posterior structures have ongoing development later in childhood. It is expected that these data will not only serve as an important and expanded normative reference reflecting development and WM maturation, but will also serve as a reference frame to identify potential structural abnormalities and diagnostic changes underlying various WM leukoencephalopathic conditions.

\section{ACKNOWLEDGMENTS}

This study was supported in part by the Robert L. McLaurin, MD, Faculty Development Scholarship in Neurosurgery at Cincinnati Children's Hospital Medical Center.
Disclosures: A. Cancelliere-RELATED: Grant: Robert L. McLaurin, MD, Faculty Development Scholarship in Neurosurgery. ${ }^{*}$ E. Air-OTHER RELATIONSHIPS: Lectured for St. Jude Medical and received fellowship support from Medtronic, both in neuromodulation (not hydrocephalus). S. Holland-RELATED: Grant: McLaurin Faculty Development Scholarship. Comments: Private foundation donation to Department of Neurosurgery in support of this work; UNRELATED: Board Membership: National Institutes of Health. Comments: Advisory Board to University of Chicago on NICHDfunded program project grant; Consultancy: Mayo Clinic, Emory University. Comments: Consulting fees for MR physics consulting unrelated to the work in the current publication. W. Yuan-RELATED: Grant: Robert L. McLaurin, MD, Faculty Development Scholarship in Neurosurgery at Cincinnati Children's Hospital Medical Center.* (*Funds paid to institution.)

\section{REFERENCES}

1. Schmithorst VJ, Wilke M, Dardzinski BJ, et al. Cognitive functions correlate with white matter architecture in a normal pediatric population: a diffusion tensor MRI study. Hum Brain Mapp 2005;26:139-47

2. Bava S, Thayer R, Jacobus J, et al. Longitudinal characterization of white matter maturation during adolescence. Brain Res 2010;1327: $38-46$

3. Sherman DL, Brophy PJ. Mechanisms of axon ensheathment and myelin growth. Nat Rev Neurosci 2005;6:683-90

4. Mukherjee P, Miller JH, Shimony JS, et al. Diffusion-tensor MR imaging of gray and white matter development during normal human brain maturation. AJNR Am J Neuroradiol 2002;23:1445-56

5. Beaulieu C. The basis of anisotropic water diffusion in the nervous system - a technical review. NMR Biomed 2002;15:435-55 
6. Neil J, Miller J, Mukherjee P, et al. Diffusion tensor imaging of normal and injured developing human brain-a technical review. NMR Biomed 2002;15:543-52

7. Wimberger DM, Roberts TP, Barkovich AJ, et al. Identification of "premyelination" by diffusion-weighted MRI. J Comput Assist Tomogr 1995;19:28-33

8. Hermoye L, Saint-Martin C, Cosnard G, et al. Pediatric diffusion tensor imaging: normal database and observation of the white matter maturation in early childhood. Neuroimage 2006;29:493-504

9. Huang $\mathrm{H}$, Zhang J, Wakana $\mathrm{S}$, et al. White and gray matter development in human fetal, newborn and pediatric brains. Neuroimage 2006;33:27-38

10. Gao W, Lin W, Chen Y, et al. Temporal and spatial development of axonal maturation and myelination of white matter in the developing brain. AJNR Am J Neuroradiol 2009;30:290-96

11. Hasan KM, Kamali A, Iftikhar A, et al. Diffusion tensor tractography quantification of the human corpus callosum fiber pathways across the lifespan. Brain Res 2009;1249:91-100

12. Ben Bashat D, Ben Sira L, Graif M, et al. Normal white matter development from infancy to adulthood: comparing diffusion tensor and high $\mathbf{b}$ value diffusion weighted MR images. J Magn Reson Imaging 2005;21:503-11

13. Peng H, Orlichenko A, Dawe RJ, et al. Development of a human brain diffusion tensor template. Neuroimage 2009;46:967-80

14. Yuan W, Holland SK, Jones BV, et al. Characterization of abnormal diffusion properties of supratentorial brain tumors: a preliminary diffusion tensor imaging study. J Neurosurg Pediatr 2008;1:263-69

15. Yuan W, Mangano FT, Air EL, et al. Anisotropic diffusion properties in infants with hydrocephalus: a diffusion tensor imaging study. AJNR Am J Neuroradiol 2009;30:1792-98

16. Sun $M$, Yuan W, Hertzler DA, et al. Diffusion tensor imaging findings in young children with benign external hydrocephalus differ from the normal population. Childs Nerv Syst 2012;28:199-208

17. Barnea-Goraly N, Menon V, Eckert M, et al. White matter development during childhood and adolescence: a cross-sectional diffusion tensor imaging study. Cereb Cortex 2005;15:1848-54

18. Mukherjee P, Miller JH, Shimony JS, et al. Normal brain maturation during childhood: developmental trends characterized with diffusion-tensor MR imaging. Radiology 2001;221:349-58

19. Lobel U, Sedlacik J, Gullmar D, et al. Diffusion tensor imaging: the normal evolution of ADC, RA, FA, and eigenvalues studied in multiple anatomical regions of the brain. Neuroradiology 2009;51: 253-63

20. Bonekamp D, Nagae LM, Degaonkar M, et al. Diffusion tensor imaging in children and adolescents: reproducibility, hemispheric, and age-related differences. Neuroimage 2007;34:733-42

21. Saksena S, Husain N, Malik GK, et al. Comparative evaluation of the cerebral and cerebellar white matter development in pediatric age group using quantitative diffusion tensor imaging. Cerebellum 2008;7:392-400

22. Morriss MC, Zimmerman RA, Bilaniuk LT, et al. Changes in brain water diffusion during childhood. Neuroradiology 1999;41:929-34

23. Basser PJ, Pierpaoli C. A simplified method to measure the diffusion tensor from seven MR images. Magn Reson Med 1998;39:928-34
24. Air EL, Yuan W, Holland SK, et al. Longitudinal comparison of preand postoperative diffusion tensor imaging parameters in young children with hydrocephalus. J Neurosurg Pediatr 2010;5:385-91

25. Brody BA, Kinney HC, Kloman AS, et al. Sequence of central nervous system myelination in human infancy. I. An autopsy study of myelination. J Neuropathol Exp Neurol 1987;46:283-301

26. Kinney HC, Karthigasan J, Borenshteyn NI, et al. Myelination in the developing human brain: biochemical correlates. Neurochem Res 1994;19:983-96

27. Kinney HC, Brody BA, Kloman AS, et al. Sequence of central nervous system myelination in human infancy. II. Patterns of myelination in autopsied infants. J Neuropathol Exp Neurol 1988;47:217-34

28. Minkowski A. Council for International Organizations of Medical Sciences, France. Délégation générale à la recherche scientifique et technique. [from old catalog]. Regional development of the brain in early life: a symposium organized by the Council for International Organizations of Medical Sciences. Oxford, Edinburgh: Blackwell Scientific; 1967

29. Dubois J, Dehaene-Lambertz G, Perrin M, et al. Asynchrony of the early maturation of white matter bundles in healthy infants: quantitative landmarks revealed noninvasively by diffusion tensor imaging. Hum Brain Mapp 2008;29:14-27

30. Schneider JF, Il'yasov KA, Hennig J, et al. Fast quantitative diffusiontensor imaging of cerebral white matter from the neonatal period to adolescence. Neuroradiology 2004;46:258-66

31. Lebel C, Walker L, Leemans A, et al. Microstructural maturation of the human brain from childhood to adulthood. Neuroimage 2008;40:1044-55

32. Rollins NK, Glasier P, Seo Y, et al. Age-related variations in white matter anisotropy in school-age children. Pediatr Radiol 2010;40: 1918-30

33. Ono J, Harada K, Takahashi M, et al. Differentiation between dysmyelination and demyelination using magnetic resonance diffusional anisotropy. Brain Res 1995;671:141-48

34. Le Bihan D. Looking into the functional architecture of the brain with diffusion MRI. Nat Rev Neurosci 2003;4:469-80

35. Takahashi M, Ono J, Harada K, et al. Diffusional anisotropy in cranial nerves with maturation: quantitative evaluation with diffusion MR imaging in rats. Radiology 2000;216:881-85

36. Giorgio A, Watkins KE, Chadwick M, et al. Longitudinal changes in grey and white matter during adolescence. Neuroimage 2010;49: $94-103$

37. McGraw P, Liang L, Provenzale JM. Evaluation of normal agerelated changes in anisotropy during infancy and childhood as shown by diffusion tensor imaging. AJR Am J Roentgenol 2002;179: 1515-22

38. Yuan W, Deren KE, McAllister JP 2nd, et al. Diffusion tensor imaging correlates with cytopathology in a rat model of neonatal hydrocephalus. Cerebrospinal Fluid Res 2010;7:19

39. Yuan W, McAllister JP 2nd, Lindquist DM, et al. Diffusion tensor imaging of white matter injury in a rat model of infantile hydrocephalus. Childs Nerv Syst 2012;28:47-54 